\title{
Monocyte-to-high density lipoprotein ratio (MHR) can predict the significance of angiographically intermediate coronary lesions iे $^{2}$
}

\author{
Ahmet Korkmaz ${ }^{\mathrm{a}, *}$, Mevlut Demir $^{\mathrm{a}}$, Sefa Unal ${ }^{\mathrm{b}}$, Abdulkadir Yildiz $^{\mathrm{c}}$, Burcu Ozyazgan $^{\mathrm{a}}$, Bekir Demirtas ${ }^{\mathrm{a}}$, \\ Ozgul Ucar Elalmis ${ }^{\text {a }}$, Mehmet Ileri ${ }^{a}$, Umit Guray ${ }^{\text {a }}$ \\ a Ankara Numune Training and Research Hospital, Department of Cardiology, Ankara, Turkey \\ b Turkey Yuksek Ihtisas Hospital, Department of Cardiology, Ankara, Turkey \\ c Bezmialem Vakif University Hospital, Department of Cardiology, Istanbul, Turkey
}

\section{A R T I C L E I N F O}

\section{Article history:}

Received 19 April 2017

Received in revised form 10 May 2017

Accepted 15 May 2017

Available online 17 May 2017

\section{Keywords:}

Monocyte to HDL ratio

Fractional flow reserve

Coronary artery stenosis

Plateletcrit

\begin{abstract}
A B S T R A C T
Purpose: Monocyte-high density lipoprotein ratio (MHR) has recently emerged as a marker of inflammation and oxidative stress in the cardiovascular disease. We aimed to investigate whether baseline MHR is associated with functional significance of intermediate coronary artery lesions.

Methods: Three hundred and one consecutive patients, 215 males and 86 females, who underwent fractional flow reserve (FFR) measurement for angiographically intermediate coronary stenosis (40-70\% in quantitative coronary analysis) in the left anterior descending coronary artery were enrolled into the study. An FFR value of $\leq 0.80$ was accepted for hemodynamic significance.

Results: Of the 301 patients, 115 (38.2\%) exhibited significant functional stenosis (FFR $\leq 0.80)$ in the FFR measurement. Patients with hemodynamically significant lesions had higher MHR values $(11.6 \pm 3.3$ vs. $12.6 \pm 2.5 \mathrm{p}=$ $0.003)$.In stepwise multivariate logistic regression analysis, total cholesterol $(\mathrm{OR}=1.008,95 \% \mathrm{CI}=1.002-1.013$, $\mathrm{p}<0.010$ ), plateletcrit $(\mathrm{OR}=1.310,95 \% \mathrm{CI}=1.097-1.564, \mathrm{p}=0.013)$ and $\mathrm{MHR}(\mathrm{OR}=2.993,95 \% \mathrm{CI}=1.365-$ $6.561, \mathrm{p}=0.008$ ) were independent predictors of significant functional stenosis. An MHR value of $12.1 \mathrm{had} 65 \%$ sensitivity and 55\% specificity for prediction of hemodynamically significant coronary artery stenosis.

Conclusions: Increased MHR values were associated with functional significance of angiographically intermediate coronary artery stenosis.
\end{abstract}

(C) 2016 The Society of Cardiovascular Academy. Production and hosting by Elsevier B.V. This is an open access article under the CC BY-NC-ND license (http://creativecommons.org/licenses/by-nc-nd/4.0/).

\section{Introduction}

Coronary angiography is one of the principal methods used for diagnosing and assessing coronary artery lesions. ${ }^{1}$ However, qualitative evaluation of coronary artery stenosis with coronary angiography is not always reliable and the visible anatomic stenosis may not be hemodynamically significant. FFR measurement is a well-established method for functional assessment of lesion severity, which involves the measurement of a coronary artery lesion's hemodynamic significance when the coronary artery stenosis is particularly between 40 and 70\% (i.e. at an intermediate level). ${ }^{2}$

Inflammation, oxidative stress, platelet activation and endothelial dysfunction assume an important role in both the development and progression of atherosclerosis. ${ }^{3,4}$ Monocytes and macrophages are the main types of cells that secrete proinflammatory cytokines, which

\footnotetext{
is Peer review under responsibility of The Society of Cardiovascular Academy.

* Corresponding author.

E-mail address: drahmtkrkmz07@gmail.com (A. Korkmaz).
}

play a central role in in the pathogenesis of atherosclerosis. ${ }^{4}$ Studies have shown that the adverse effects of low-density lipoproteins (LDLs) on endothelial cells can be limited by high-density lipoprotein cholesterol (HDL-C), which prevents the oxidation of LDL. ${ }^{5-7}$ HDL-C is believed to have both antioxidant and anti-inflammatory properties. A new cardiovascular disease marker that has appeared in recent times is the monocyte to HDL-C ratio (MHR), which combines the prognostic and predictive effectiveness of two widely used and accessible laboratory parameters. ${ }^{8-10}$ The aim of the present study was to examine the relationship between MHR and the functional significance of intermediate level coronary artery stenosis evaluated with FFR measurement.

\section{Subjects and methods}

This retrospective study was conducted between January 2012 and March 2016 with a total of 301 consecutive patients, including 86 female and 215 male patients, diagnosed with single intermediategrade coronary stenosis (40-70\%, based on quantitative coronary analysis) on their left anterior descending coronary artery who were examined with fractional flow reverse (FFR) measurement. Patients who had 
undergone coronary angiography with an indication of stable angina pectoris involved in the present study. Patients with the following conditions were excluded from the study: acute coronary syndrome; moderate or severe valvular heart disease; significant arrhythmia; hemodynamic instability; second lesion at the index coronary artery; another coronary artery with $\mathrm{a} \geq 40 \%$ luminal narrowing (determined by coronary angiography); a history of previous surgical or percutaneous coronary artery intervention; acute or chronic inflammatory or infectious diseases; anemia, chronic renal failure, and malignancy.

Hospital files and records were examined to determine the patients' demographic, clinical and angiographic data. Blood samples were collected from veins within $24 \mathrm{~h}$ after hospital admission. Measurements included lipid profile, serum creatinine and complete blood count. The patients' baseline MHR was determined by dividing their monocyte count with their HDL cholesterol level. The institutional local ethics committee approved the study protocol.

FFR measurements for intermediate-grade lesions with a $40-70 \%$ stenosis rate were performed based on the cardiologists' decision and discretion. Following the intra-arterial administration of a 5000 unit heparin bolus, the coronary artery was examined by employing a guiding catheter without side holes. A 0.014 in. pressure monitoring guidewire (PrimeWire, Volcano, San Diego, Calif., USA) was positioned distally to the stenosis after calibration. A $200 \mu \mathrm{g}$ bolus of nitroglycerin was administered intracoronarily prior to FFR measurements. The patients' distal intracoronary pressure was recorded at baseline, and hyperemia was triggered by applying intracoronary adenosine at gradually increasing doses until the FFR value ceased to decrease any further. FFR was determined as the ratio between the mean distal intracoronary pressure and the mean aortic pressure, at the moment the highest level of hyperemia is observed.

An FFR value of $\leq 0.80$ was defined as functionally significant. Patients with an FFR value of $>0.80$ formed group I and patients with an FFR value of $\leq 0.80$ formed group II.

\section{Statistical analysis}

Statistical analysis was performed using the SPSS 18.0 for Windows (SPSS Inc., Chicago, IL, USA) package software. Whether the data exhibited normal distribution was determined using the Kolmogorov-Smirnov test. The chi-square test was used for comparing categorical variables, while the Mann-Whitney $U$ test or Student's t-test were used for comparing continuous variables. Percentages were used for presenting categorical variables, while mean \pm standard deviation or median (interquartile range) were used for presenting continuous variables. Possible confounding factors for the coronary artery lesions' severity were determined using the univariate and multiple logistic regression analysis. The multiple regression model was used to test variables in the univariate regression analysis that has p values below 0.10 . To determine optimum MHR cutoff value for predicting the hemodynamic significance of the coronary artery stenosis, the receiver-operating characteristic (ROC) curve was employed. Statistical significance level was set at $<0.05$.

\section{Results}

The baseline characteristics of the patients are shown in Table 1. Among the 301 patients included into the study, 115 (38.2\%) were found to have significant functional stenosis. Group II has a lower mean age than group I ( $58 \pm 12$ vs. $61 \pm 11$ years, $\mathrm{p}=0.007$ ). While group II had a higher ratio of male patients, the difference between the two groups was not significant ( $68 \%$ vs. $77 \%, p=0.072$ ). There were also no statistically significant differences with regards to the coronary risk factors, including smoking, hyperlipidemia, hypertension and diabetes mellitus (Table 1 ).

The laboratory parameters of two groups are reported in Table 2. Group II had higher level of total cholesterol (203 \pm 46 vs. $188 \pm$ $42 \mathrm{mg} / \mathrm{dL}, \mathrm{p}=0.004)$. LDL-C level was also higher in group II, although
Table 1

Basal characteristics of the study groups.

\begin{tabular}{llll}
\hline Variable & $\begin{array}{l}\text { Insignificant FFR } \\
(\mathrm{n}=186)\end{array}$ & $\begin{array}{l}\text { Significant FFR } \\
(\mathrm{n}=115)\end{array}$ & p value \\
\hline Age, year & $61 \pm 11$ & $58 \pm 12$ & $\mathbf{0 . 0 0 7}$ \\
Basal FFR & $0.93 \pm 0.03$ & $0.86 \pm 0.08$ & $<\mathbf{0 . 0 0 1}$ \\
Smoking, $\mathrm{n}(\%)$ & $91(49)$ & $55(48)$ & 0.853 \\
Male gender, n (\%) & $126(68)$ & $89(77)$ & 0.072 \\
Diabetes mellitus, n, \% & $56(30)$ & $30(26)$ & 0.453 \\
Hypertension, n, \% & $69(37)$ & $42(37)$ & 0.920 \\
Hyperlipidemia, n, \% & $51(28)$ & $24(21)$ & 0.198 \\
\hline
\end{tabular}

FFR - fractional flow reserve. Data are expressed as mean + standard deviation for normally distributed parametric variables, median (interquartile range) for non-parametric variables and percentage for categorical variables.

the difference was not statistically significant $(125 \pm 39$ vs. $117 \pm$ $38 \mathrm{mg} / \mathrm{dL}, \mathrm{p}=0.078$ ). Group II exhibited higher monocyte count (50 (40-56) vs. 55 (45-58), $\mathrm{p}=0.004)$, plateletcrit $(0.21 \pm 0.01$ vs. $0.20 \pm 0.01, \mathrm{p}=0.002)$ and $\mathrm{MHR}(12.67 \pm 2.59$ vs. $11.65 \pm 3.33, \mathrm{p}=$ 0.003). Group I had a higher lymphocyte-to-monocyte ratio (LMR), although this difference was not statistically significant $0.044(0.034-$ $0.057)$ vs. $0.042(0.030-0.054), \mathrm{p}=0.227)$. Other laboratory data did not differ significantly between the two groups (Table 2 ).

Univariate and multiple logistic regression analysis indicated that MHR ( OR $=3.401,95 \% \mathrm{CI}=1.378-8.391, \mathrm{p}=0.008)$, plateletcrit $(\mathrm{OR}=1.276,95 \% \mathrm{CI}=1.052-1.549, \mathrm{p}=0.013)$ and total cholesterol ( $\mathrm{OR}=1.025,95 \% \mathrm{CI}=1.006-1.044, \mathrm{p}=0.010)$ are independent predictors of significant functional stenosis (Table 3). ROC analysis revealed that an MHR value of 12.1 had a specificity of $55 \%$ and a sensitivity of $65 \%$ in predicting hemodynamically significant coronary artery stenosis (Fig. 1).

\section{Discussion}

In this study, MHR levels were independently associated with functionally significant coronary artery lesions assessed with FFR

Table 2

Comparison of laboratory parameters between patients with hemodynamically significant coronary artery lesions and patients with hemodynamically non-significant coronary artery lesions.

\begin{tabular}{llll}
\hline Variable & $\begin{array}{l}\text { Insignificant FFR } \\
(\mathrm{n}=186)\end{array}$ & $\begin{array}{l}\text { Significant FFR } \\
(\mathrm{n}=115)\end{array}$ & $\mathrm{p}$ value \\
\hline Glucose, $\mathrm{mg} / \mathrm{dL}$ & $110(94-153)$ & $112(94-147)$ & 0.838 \\
Urea, $\mathrm{mg} / \mathrm{dL}$ & $34(28-39)$ & $34(27-40)$ & 0.951 \\
Creatinine, $\mathrm{mg} / \mathrm{dL}$ & $0.91(0.79-1.04)$ & $0.93(0.80-1.03)$ & 0.678 \\
Total cholesterol, $\mathrm{mg} / \mathrm{dL}$ & $188 \pm 42$ & $203 \pm 46$ & $\mathbf{0 . 0 0 4}$ \\
Trygliceride, $\mathrm{mg} / \mathrm{dL}$ & $146(107-195)$ & $154(112-242)$ & 0.122 \\
HDL-C, mg/dL & $42(35-50)$ & $41(37-45)$ & 0.495 \\
LDL-C, mg/dL & $117 \pm 38$ & $125 \pm 39$ & 0.078 \\
WBC count, $\times 10^{9} / \mathrm{L}$ & $8.5 \pm 2.7$ & $8.9 \pm 2.3$ & 0.233 \\
Neutrophile count, $\times 10^{9} / \mathrm{L}$ & $5.46 \pm 2.41$ & $5.81 \pm 2.03$ & 0.199 \\
Lymphocyte count, $\times 10^{9} / \mathrm{L}$ & $2.22 \pm 0.84$ & $2.25 \pm 0.83$ & 0.709 \\
Monocyte count, $\times 10^{12} / \mathrm{L}$ & $50(40-56)$ & $55(45-58)$ & $\mathbf{0 . 0 0 4}$ \\
Eosinophile count, $\times 10^{12} / \mathrm{L}$ & $23(11-38)$ & $30(20-40)$ & 0.054 \\
Hb, gr/dL & $14.2(13.0-15.4)$ & $14.5(13.3-15.4)$ & 0.241 \\
RDW & $13.7(13.0-14.6)$ & $13.5(13.1-14.3)$ & 0.212 \\
Platelet count, $\times 10^{9} / \mathrm{L}$ & $254 \pm 62$ & $247 \pm 65$ & 0.369 \\
MPV, fL & $8.4 \pm 0.6$ & $8.4 \pm 1.4$ & 0.641 \\
Platecrit $(\mathrm{PCT})$ & $0.20 \pm 0.01$ & $0.21 \pm 0.01$ & $\mathbf{0 . 0 0 2}$ \\
PDW & $16.9(16.3-18.0)$ & $16.8(16.2-17.5)$ & 0.338 \\
NLR & $2.39(1.75-3.19)$ & $2.56(1.74-3.54)$ & 0.196 \\
PLR & $121(93-151)$ & $117(91-155)$ & 0.369 \\
LMR & $0.044(0.034-0.057)$ & $0.042(0.030-0.050)$ & 0.227 \\
MHR & $11.65 \pm 3.33$ & $12.67 \pm 2.59$ & $\mathbf{0 . 0 0 3}$ \\
\hline
\end{tabular}

FFR - fractional flow reserve; HDL - high-density lipoprotein; LDL - low-density lipoprotein; MPV - mean platelet volume; NLR - neutrophil-to-lymphocyte ratio; PLR - plateletto-lymphocyte ratio; RDW - red cell distribution width; WBC - white blood cell; LMR lymphocyte-to-monocyte ratio; MHR - monocyte count -to -HDL-C ratio. Data are expressed as mean \pm standard deviation for normally distributed parametric variables and median (interquartile range) for non-parametric variables.

Bold values indicate significance at $\mathrm{p}<0.05$. 
Table 3

Univariate and multiple logistic regression analyses performed to find out possible confounding factors of hemodynamically significant coronary artery stenosis.

\begin{tabular}{|c|c|c|c|c|c|c|}
\hline \multirow[t]{2}{*}{ Variable } & \multicolumn{3}{|c|}{ Univariate analysis } & \multicolumn{3}{|c|}{ Multivariate analysis } \\
\hline & $\begin{array}{l}\text { Odds } \\
\text { ratio }\end{array}$ & $95 \% \mathrm{CI}$ & $\mathrm{p}$ value & Odds ratio & $95 \% \mathrm{CI}$ & $\mathrm{p}$ value \\
\hline T chol & 1.008 & $1.002-1.013$ & 0.005 & 1.025 & $1.006-1.044$ & 0.010 \\
\hline Age & 0.971 & $0.950-0.992$ & 0.008 & 0.984 & $0.961-1.008$ & 0.193 \\
\hline LDL-C & 1.006 & $0.999-1.012$ & 0.079 & 0.984 & $0.967-1.000$ & 0.053 \\
\hline PCT & 1.310 & $1.097-1.564$ & 0.003 & 1.276 & $1.052-1.549$ & 0.013 \\
\hline MHR & 2.993 & $1.365-6.561$ & 0.006 & 3.401 & $1.378-8.391$ & 0.008 \\
\hline Male gender & 1.603 & $0.939-2.738$ & 0.084 & 1.436 & $0.788-2.615$ & 0.237 \\
\hline
\end{tabular}

T chol - total cholesterol; LDL-C - low-density lipoprotein cholesterol; PCT -plateletcrit; MHR - monocyte count -to -HDL-C ratio.

Bold values indicate significance at $\mathrm{p}<0.05$.

measurement. In addition, total cholesterol and plateletcrit remained independent predictors of functional significance on multivariate analysis in the current study.

Oxidative stress and inflammation are central mechanisms in the development and progress of atherosclerosis. ${ }^{3,4,9}$ Monocytes play a particularly important role in these processes. ${ }^{4,5}$ The interaction of activated monocytes with activated or damaged endothelium leads to the overexpression of adhesion molecules and proinflammatory cytokines, such as the intercellular adhesion molecule-1, the vascular cell adhesion molecule- 1 , and the monocyte chemotactic protein- 1 ligand. ${ }^{4,11,12}$ Monocytes then differentiate into the macrophage cells, which absorb oxidized LDL cholesterol molecules, forming harmful foamy cells in the process. ${ }^{12}$ On the contrary, HDL-cholesterol molecules reduce macrophage accumulation and promote removal of oxidized cholesterol from arterial wall. ${ }^{13-}$ ${ }^{15}$ Recent studies also showed that HDL-cholesterol can inhibit monocyte activation, adhesiveness, and inflammation. ${ }^{5-7}$ In addition to its antioxidative and antiinflammatory effects, HDL also increases the expression of nitric oxide synthase in endothelial tissues and promotes vasorelaxation. ${ }^{16}$ Higher HDL levels are known to provide protection against atherosclerosis, and are associated with better prognosis among patients with atherosclerosis. ${ }^{17}$ Therefore, HDL-C counteracts the predominantly prooxidant and proinflammatory effects of monocytes.

MHR is a new and promising marker that indicates the inflammatory state of the patient. Based on the current literature, we assumed that higher MHR values might serve as an effective predictor of functional significance of coronary artery stenosis.

Qualitative evaluation of coronary artery stenosis with coronary angiography is not always reliable and the visible anatomic stenosis may not be hemodynamically significant. ${ }^{2,18}$ FFR measurement is a wellestablished method for functional assessment of lesion severity. FFR technique is based on pressure decline between proximal and distal parts of the stenosis after obtaining maximal hyperemia and linearly related to maximum blood flow to the myocardium supplied by the respective coronary artery. Thus, in this study FFR measurement was utilized to determine the functional significance of coronary lesion severity and its relationship with the MHR level.

MHR is a newly discovered marker for oxidative stress and inflammation. Kanbay et al. previously suggested that in patients with chronic kidney disease, high MHR values are linked with worse cardiovascular prognosis. ${ }^{8}$ Another study reported that high MHR values are correlated with a greater severity and occurrence of isolated coronary artery ectasia. ${ }^{10}$ Kundi et al. demonstrated, based on SYNTAX score assessments, a relationship between higher MHR and greater coronary atherosclerosis severity. ${ }^{19}$ Cetin et al. demonstrated that in patients with acute coronary syndrome MHR was an independent predictor of stent thrombosis and severity of coronary artery disease as well as future cardiovascular events. ${ }^{20,21}$ Also in a study of Cicek et al., MHR was found to be significantly associated with both short- and long term mortality in patients with ST elevation myocardial infarction who underwent primary percutaneous coronary intervention. ${ }^{22}$

In our study, we found that MHR is significantly higher in patients with hemodynamically significant lesions assessed by FFR (FFR $\leq$ 0.80). There are some possible explanations of our findings. First of all, it was clearly shown that patients with hemodynamically significant coronary lesions have a poor prognosis and increased MHR has been closely linked with worse outcomes. ${ }^{8,20-22}$ Secondly, higher MHR can

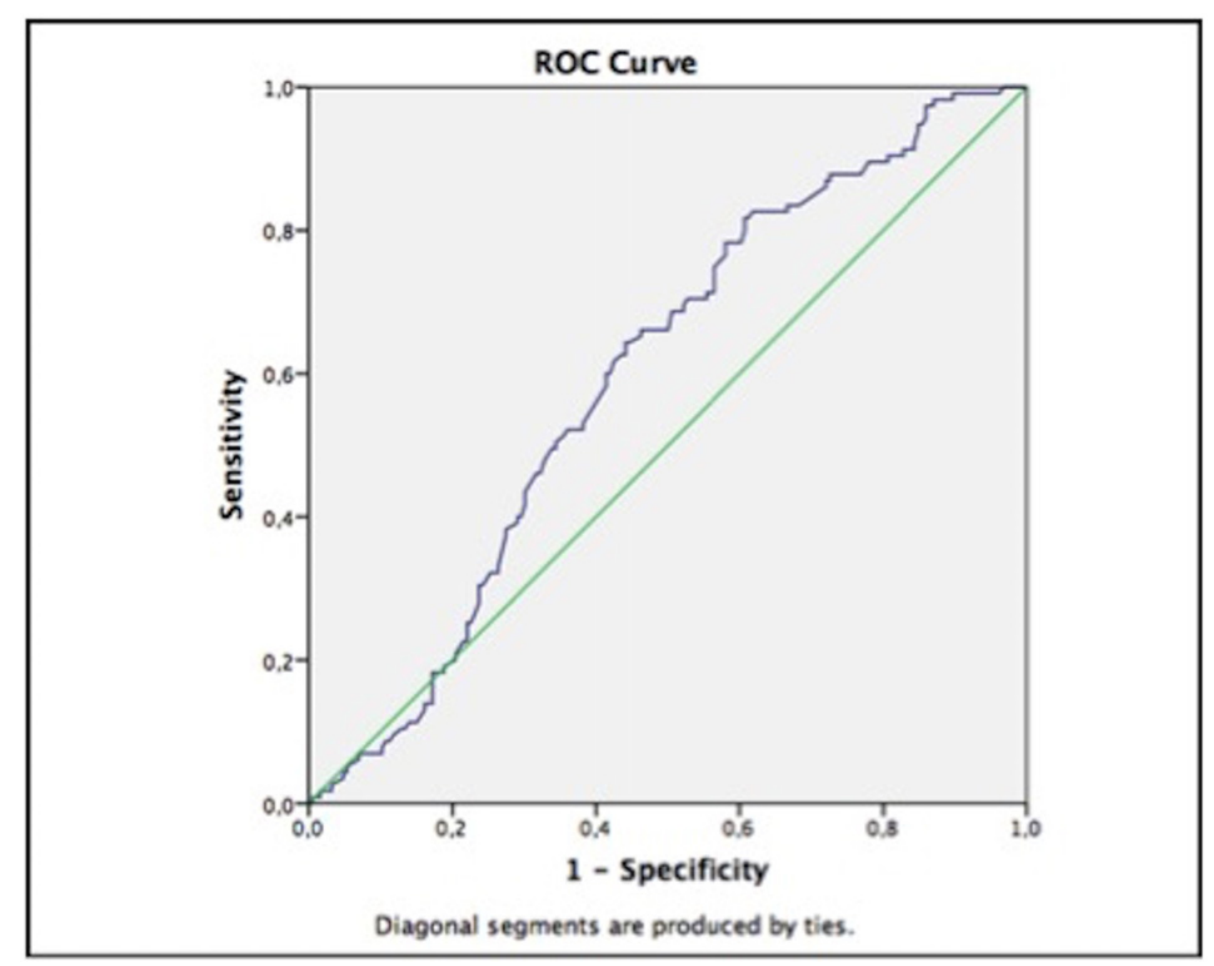

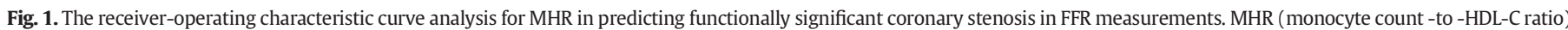
value of 12.1 had $65 \%$ sensitivity and $55 \%$ specificity for prediction of hemodynamically significant coronary artery stenosis. AUC: $0.598,95 \% \mathrm{CI}=0.534-0.662$, $\mathrm{p}=0.004$. 
be viewed as a reaction to coronary artery lesions of greater severity. Based on this view, we consider that ischemias can lead to various inflammatory responses that alter WBC distribution and count. There is evidence suggesting that higher monocyte levels are linked with the development of proinflammatory and atherosclerotic responses. ${ }^{3,4,9,11-12}$ It is known that HDL-C molecules can inhibit the activation and movement of monocytes. ${ }^{13-15}$ The observation that coronary artery disease patients with high SYNTAX levels $(\geq 23$ ) tend to have with increased MHR values is in line with the present study's findings that increased MHR may serve as a predictor of functional significance in patient with intermediate-grade coronary artery stenoses. ${ }^{19,23}$ In addition, previous studies indicate that severe CAD with good collaterals tend to have high monocyte counts, while those which poorer collaterals have lower HDL levels. This finding is also in agreement with the results of our study. ${ }^{24-26}$ Considering that the degree and duration of coronary stenosis is the one of the most important determinants of coronary collateral development, it can be said that MHR values can help predict significant coronary stenosis that leads to myocardial ischemia.

In contrast to other studies, we determined during the multivariate analysis that plateletcrit and total cholesterol are both independent predictors for significant functional stenosis. These findings are in line with the known pathogenesis of coronary atherosclerosis. But there is insignificant correlation between functional stenosis, MHR and LDL-Cholesterol. Previous similar studies showed that there was not statistically significant difference between lipid biomarkers and functional coronary stenosis severity in stable coronary artery disease. ${ }^{27-29}$ The difference in results between our work and other studies, can be explained by the he effects of antihyperlipidemic treatment; and genetic, environmental (including obesity, physical inactivity, and cigarette smoking), and unmeasured variables.

Studies have demonstrated that the platelet-to-lymphocyte ratio (PLR) and the neutrophil-to-lymphocyte ratio (NLR) are both significant markers of inflammation, and that they are linked with a variety of cardiovascular diseases. ${ }^{30-32}$ In two studies similar to our own on the FFR, red cell distribution width (RDW) and NLR showed close relationship with the functional significance of intermediate-grade coronary artery stenoses. ${ }^{28-29}$ However, in our study, it was observed that PLR, NLR and RDW did not function as independent predictors hemodynamically severe coronary artery stenosis. Similarly, Sels et al.'s study failed to demonstrate any relationship of FFR with inflammatory markers - such as the tumor necrosis factor, interleukin-6, interleukin- 8 and WBC count. ${ }^{33}$ These different results might be explained with differences in patient characteristics and numbers in different studies. Also, these differences might also be accounted by the different approaches of researchers towards coronary stenotic lesions (e.g. percutaneous, medical and sintigraphy approaches).

The evaluation of platelet indices such as platelet counts, mean platelet volume (MPV), platelet distribution width (PDW) and plateletcrit (PCT) is easy and practical and can play a role in the onset and progression of atherosclerosis. ${ }^{34}$ In our study, only higher PCT is independent predictors for significant functional stenosis but there is weak correlation. There are few studies investigating the relationship between PCT and coronary artery disease in the literature. Previous studies suggested that high PCT values on admission are independently associated with long-term adverse outcomes in patients with ST-segment elevation myocardial infarction (STEMI), non STEMI (NSTEMI), coronary slow flow phenomenon and saphenous vein grafting disease. ${ }^{35-38}$ In the light of these findings PCT may show correlation with significant functional stenosis of angiographically intermediate coronary lesions, but this may be a coincidence arising from the coexistence of atherosclerosis severity and not a causal relationship.

\section{Study limitations}

The present study had a number of limitations. First of all, the study has a retrospective design with limited number of patients. Secondly, many other important markers of inflammation - such as the hs-CRP - were not used in this study (although it is certainly unfeasible to conduct a study that can include and examine all types of inflammatory markers). Thirdly, our study analyses were based on a single MHR values; in other words, we did not follow temporal changes and variations in MHR. Finally our study does not provide a mechanistic explanation for the effect of specific monocyte subsets on the severity of CAD and MHR

\section{Conclusion}

The present study demonstrated that in FFR assessments, MHR values exhibit an independent relationship with the functional significance of angiographically intermediate coronary artery stenosis. As they are based on commonly used and low cost parameter found in lipid panels and complete blood counts, MHR values can be readily determined to predict the likelihood of hemodynamically significant coronary artery stenosis in clinical settings. Nevertheless, the results of this study should be further expanded and confirmed through studies involving larger samples and prospective designs.

\section{References}

1. Montalescot G, Sechtem U, Achenbach S, et al. 2013 ESC guidelines on the management of stable coronary artery disease: the task force on the management of stable coronary artery disease of the European Society of Cardiology. Eur Heart J 2013;34: 2949-3003.

2. Tobis J, Azarbal B, Slavin L. Assessment of intermediate severity coronary lesions in the catheterization laboratory. J Am Coll Cardiol 2007;49:839-848.

3. Hansson GK. Inflammation, atherosclerosis, and coronary artery disease. $\mathrm{N} \mathrm{Engl} \mathrm{J} \mathrm{Med}$ 2005;352:1685-1695

4. Hilgendorf I, Swirski FK, Robbins CS. Monocyte fate in atherosclerosis. Arterioscler Thromb Vasc Biol 2015 Mar;35:272-279.

5. Hessler JR, Robertson Jr AL, Chisolm III GM. LDL-induced cytotoxicity and its inhibition by HDL in human vascular smooth muscle and endothelial cells in culture. Atherosclerosis 1979;32:213-229.

6. Li XP, Zhao SP, Zhang XY, et al. Protective effect of high density lipoprotein on endothelium-dependent vasodilatation. Int J Cardiol 2000;73:231-236.

7. Parthasarathy S, Barnett J, Fong LG. High-density lipoprotein inhibits the oxidative modification of low-density lipoprotein. Biochim Biophys Acta 1990;1044:275-283.

8. Kanbay M, Solak Y, Unal HU, et al. Monocyte count/HDL cholesterol ratio and cardiovascular events in patients with chronic kidney disease. Int Urol Nephrol 2014;46: 1619-1625.

9. Canpolat U, Çetin EH, Cetin S, et al. Association of monocyte-to-HDL cholesterol ratio with slow coronary flow is linked to systemic inflammation. Clin Appl Thromb Hemost 2016 Jul:22:476-482.

10. Kundi H, Gok M, Kiziltunc E, et al. Relation between monocyte to high-density lipoprotein cholesterol ratio with presence and severity of isolated coronary artery ectasia. Am J Cardiol 2015;116:1685-1689.

11. Mestas J, Ley K. Monocyte-endothelial cell interactions in the development of atherosclerosis. Trends Cardiovasc Med 2008;18:228-232

12. Woollard KJ, Geissmann F. Monocytes in atherosclerosis: subsets and functions. Nat Rev Cardiol 2010;7:77-86.

13. Murphy AJ, Woollard KJ. High-density lipoprotein: a potent inhibitor of inflammation. Clin Exp Pharmacol Physiol 2010;37:710-718.

14. Murphy AJ, Chin-Dusting JP, Sviridov D, et al. The anti-inflammatory effects of high density lipoproteins. Curr Med Chem 2009;16:667-675.

15. Yvan-Charvet L, Pagler T, Gautier EL, et al. ATP-binding cassette transporters and HDL suppress hematopoietic stem cell proliferation. Science 2010;328:1689-1693.

16. Kuvin JT, Ramet ME, Patel AR, et al. A novel mechanism for the beneficial vascular effects of high-density lipoprotein cholesterol: enhanced vasorelaxation and increased endothelial nitric oxide synthase expression. Am Heart J 2002;144:165-172.

17. Van de Woestijne AP, van der Graaf Y, Liem A-H, et al, SMART Study Group. Low high-density lipoprotein cholesterol is not a risk factor for recurrent vascular events in patients with vascular disease on intensive lipid-lowering medication. J Am Coll Cardiol 2013 Nov 12;62:1834-1841.

18. Fischer JJ, Samady H, McPherson JA, et al. Comparison between visual assessment and quantitative angiography versus fractional flow reserve for native coronary narrowings of moderate severity. Am J Cardiol 2002;90:210-215.

19. Kundi H, Kiziltunc E, Cetin M, et al. Association of monocyte/HDL-C ratio with SYNTAX scores in patients with stable coronary artery disease. Herz 2016 Jan 11.

20. Cetin EHO, Cetin MS, Canpolat U, et al. Monocyte/HDL cholesterol ratio predicts the definite stent thrombosis after primary percutaneous coronary intervention for ST-segment elevation myocardial infarction. Biomark Med 2015:9:967-977.

21. Cetin MS, Ozcan Cetin EH, Kalender E, et al. Monocyte to HDL cholesterol ratio predicts coronary artery disease severity and future major cardiovascular adverse events in acute coronary syndrome. Heart Lung Circ 2016. 
22. Çiçek G, Kundi H, Bozbay M, et al. The relationship between admission monocyte HDL-C ratio with short-term and long-term mortality among STEMI patients treated with successful primary PCI. Coron Artery Dis 2016 May;27:176-184.

23. Akboga MK, Balci KG, Maden O, et al. Usefulness of monocyte to HDL-cholesterol ratio to predict high SYNTAX score in patients with stable coronary artery disease. Biomark Med 2016 Apr;10:375-383.

24. Heil M, Ziegelhoeffer T, Pipp F, et al. Blood monocyte concentration is critical for enhancement of collateral artery growth. Am J Physiol Heart Circ Physiol 2002 Dec;283: H2411-H2419.

25. Kocaman SA, Arslan U, Tavil Y, et al. Increased circulating monocyte count is related to good collateral development in coronary artery disease. Atherosclerosis 2008 Apr:197:753-756.

26. Kadi H, Ozyurt H, Ceyhan K, et al. The relationship between high-density lipoprotein cholesterol and coronary collateral circulation in patients with coronary artery disease. J Invest Med 2012 Jun;60:808-812.

27. Muller O, Ntalianis A, Wijns W, et al. Association of biomarkers of lipid modification with functional and morphological indices of coronary stenosis severity in stable coronary artery disease. J Cardiovasc Transl Res 2013 Aug;6(4):536-544.

28. Açıkgöz SK, Açar B, Aydın S, et al. Red cell distribution width can predict the significance of angiographically intermediate coronary lesions. Med Princ Pract 2016;25: 31-35.

29. Akyel A, Yayla C, Erat M, et al. Neutrophil-to-lymphocyte ratio predicts hemodynamic significance of coronary artery stenosis. Anatol J Cardiol 2015;15:1002-1007.

30. Papa A, Emdin M, Passino C, et al. Predictive value of elevated neutrophil-lymphocyte ratio on cardiac mortality in patients with stable coronary artery disease. Clin Chim Acta 2008;395:27-31.
31. Tamhane UU, Aneja S, Montgomery D, et al. Association between admission neutrophil to lymphocyte ratio and outcomes in patients with acute coronary syndrome. Am J Cardiol 2008 Sep 15;102:653-657.

32. Kurtul A, Murat SN, Yarlioglues M, et al. Association of platelet-to lymphocyte ratio with severity and complexity of coronary artery disease in patients with acute coronary syndromes. Am J Cardiol 2014;114:972-978.

33. Sels JW, Elsenberg EH, Hoefer IE, et al. Fractional flow reserve is not associated with inflammatory markers in patients with stable coronary artery disease. PLoS One 2012;7e46356.

34. Tsiara S, Elisaf M, Jagroop IA, et al. Platelets as predictor of vascular risk: is there a practical index of platelet activity? Clin Appl Thromb Hemost 2003;9:177-190.

35. Akpinar I, Sayin MR, Gursoy YC, et al. Plateletcrit and red cell distribution width are independent predictors of the slow coronary flow phenomenon. J Cardiol 2014;63: $112-118$.

36. Akpinar I, Sayin MR, Gursoy YC, et al. Plateletcrit: a platelet marker associated with saphenous vein graft disease. Herz 2014;39:142-148.

37. Ugur M, Ayhan E, Bozbay M, et al. The independent association of plateletcrit with long-term outcomes in patients undergoing primary percutaneous coronary intervention. J Crit Care 2014;29:978-981.

38. Gul M, Uyarel H, Akgul O, et al. Long-term prognostic significance of admission plateletcrit values in patients with non-ST elevation myocardial infarction. Blood Coagul Fibrinolysis 2016 Sep;27(6):696-701. 